\title{
BIPARTITE MEDIAL CUNEIFORM AND CALCANEO-NAVICULAR COALITION IN A SINGLE INDIVIDUAL
}

\section{Anna L Watts *1, Nicholas Duncan ${ }^{2}$, Mark B Davies ${ }^{3}$.}

*1 Core Surgical Trainee, Department of Trauma and Orthopaedics, Sheffield Teaching Hospitals NHS Trust, Sheffield, UK.

2 Senior Foot and Ankle Fellow, Department of Trauma and Orthopaedics, Sheffield Teaching Hospitals NHS Trust, Sheffield, UK.

3 Consultant Trauma and Orthopaedic Surgeon, Department of Trauma and Orthopaedics, Sheffield Teaching Hospitals NHS Trust, Sheffield, UK.

\section{ABSTRACT}

Background: A bipartite medial cuneiform is a rare anatomical variation of the midfoot. Whilst it has been described in isolation there are few reports describing this variation alongside other anatomical anomalies of the foot.

Results: We are reporting the first case of a bipartite medial cuneiform in association with a calcaneonavicular coalition in a 27 year old male who presented with midfoot pain.

Conclusions: This case highlights the interesting origins of two seemingly opposing processes; demonstrating both the over-differentiation of primordial mesenchyme in-utero and separation and failure of fusion between two ossific nuclei.

KEY WORDS: Bipartite Medial Cuneiform, Tarsal coalition, Calcaneonavicular coalition.

Address for Correspondence: Anna L Watts MRCS, MBChB, Core Surgical Trainee, Department of Trauma and Orthopaedics, Sheffield Teaching Hospitals NHS Trust, Sheffield, UK.

E-Mail: Anna.lillian.watts@gmail.com

\section{Access this Article online}

\section{Quick Response code}

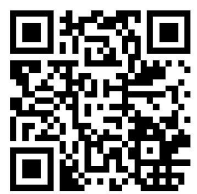

DOI: $10.16965 /$ ijar.2017.126

Web site: International Journal of Anatomy and Research ISSN 2321-4287

www.ijmhr.org/ijar.htm

Received: 27 Jan 2017

Peer Review: 28 Jan 2017

Revised: None
Accepted: 02 Mar 2017

Published (O): 31 Mar 2017

Published (P): 31 Mar 2017

\section{INTRODUCTION}

Bipartition of the medial cuneiform is a mal-segmentation defect of the midfoot in which the normal cuneiform is separated into dorsal and plantar segments [1-2], often referred to as os cuneiforme I dorsale and os cuneiforme I plantare [1,3-4].

During early embryonic development the medial cuneiform forms either from a single or two separate portions of a single cartilaginous anlage [1,4-7]. Bipartition occurs when abnormal organisation of the primordial mesenchyme leads to failure of the two separate portions merging, thus leaving a cartilaginous or fibrocartilagenous synchrondrosis between the two segments [1-2,4-5,8-10]. Cases of partial and complete bipartition have been described [1].

In partial bipartition a cleft or crease is present within the articular facet without separation into two distinct plantar and dorsal segments [11] as shown in Figure 1.

Ossification of the cuneiforms begins in the first year of life, starting with the lateral cuneiform 
and progressing medially until complete ossification occurs around the age of $3[4-5,8]$. During this time the bipartite deformity may become apparent on imaging with a characteristic 'E-Sign' [4-6], which can be seen on both sagittal MRI and CT scans as demonstrated in Figure 2.

Fig. 1: An intraoperative view of the bipartite facets of a medial cuneiform.

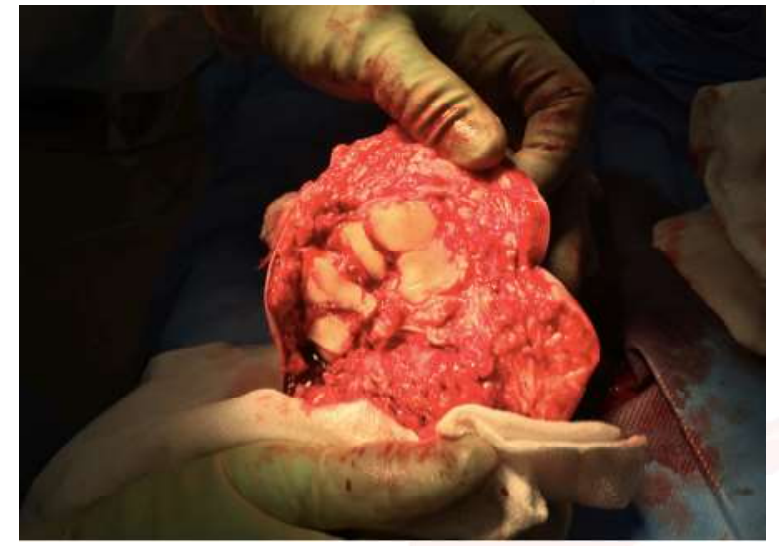

Fig. 2: A sagittal CT scan showing a characteristic 'ESign' - here a clear joint space is demonstrated between the base of the $1^{\text {st }}$ metatarsal and both the dorsal and planter segments of the bipartite medial cuneiform bones.

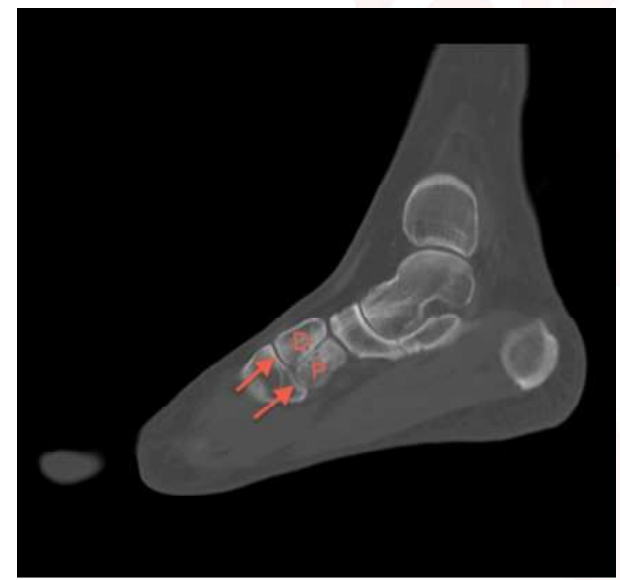

The volume of a bipartite medial cuneiform is recognised as being slightly larger than that of a normal medial cuneiform with an increased dorso-plantar height of between 5-8mm [1,5,1112]. Variations in the articular surfaces include a split facet for articulation of the plantar and dorsal segments with the first metatarsal, as well as that for the navicular [11-12]. The plantar segment receives the tendinous insertions of tibialis posterior and peroneus longus on its inferomedial and inferolateral surface respectively $[2,6]$ Tibialis anterior attaches to the superomedial surface of the dorsal segment $[2,6]$. The dorsal segment is also the site of attachment for both the dorsal and interosseous portions of the Lisfranc ligament $[2,6,8]$. The planter Lisfranc ligament extends from the plantar segment of the bipartite medial cuneiform and both attach onto the second metatarsal $[2,6,8]$.

In isolation a bipartite medial cuneiform is a rare anatomical variant. A recent meta analysis identified 57 reported cases of complete bipartition from anatomical, clinical and archaeological reports [1]._There have been relatively few studies large enough to estimate the incidence of bipartition however the three largest series quote frequencies between $0.27 \%$ and $0.31 \%[1,12]$.

A genetic predisposition for bipartite medial cuneiform has been highlighted with a high frequency of bilateral cases of both partial and complete bipartition [1,4,11]. Additionally, there is also a higher than anticipated frequency of bipartite medial cuneiform found within archaeological populations of low genetic diversity [7,9,11-12]. Pathways responsible for the normal patterning and shape of the skeleton such as Hox and Sox transcription factors alongside local regulators of mesenchyme condensation have been cited as potential genetic factors [11]. Interestingly, a meta-analysis by Burnett et al showed that within the reported cases there was a strong predominance for males with $82.5 \%$ being male opposed to just $17.5 \%$ female $[1,4]$.

A calcaneo-navicular coalition is a more common anatomical variant in which there is congenital failure of differentiation of the ossific nuclei of the calcaneum and navicu$\operatorname{lar}[13]$. The true incidence of tarsal coalitions is unknown as many are asymptomatic, however it is often quoted at $1 \%$ with calcaneo-navicular coalitions accounting for $71.4-77.2 \%$ of these [14-15]. No cases of a bipartite medial cuneiform alongside a calcaneonavicular coalition have been described in the literature.

\section{CASE REPORT}

We report a case of a 27 year old male who presented with an isolated foot injury following a falling from his bicycle. Clinical examination revealed swelling and bruising over the dorsum of the foot and tenderness to palpation over the dorso-lateral aspect of the navicular. The patient 
was also noted to have marked pes planovalgus with a rigid subtalar joint. Plain radiographs, as shown in Figure 3 were taken, which demonstrated a fracture through a calcaneo-navicular coalition corresponding to the area of maximal tenderness.

Fig. 3: AP radiograph showing a fracture through the calcaneonavicular coalition.

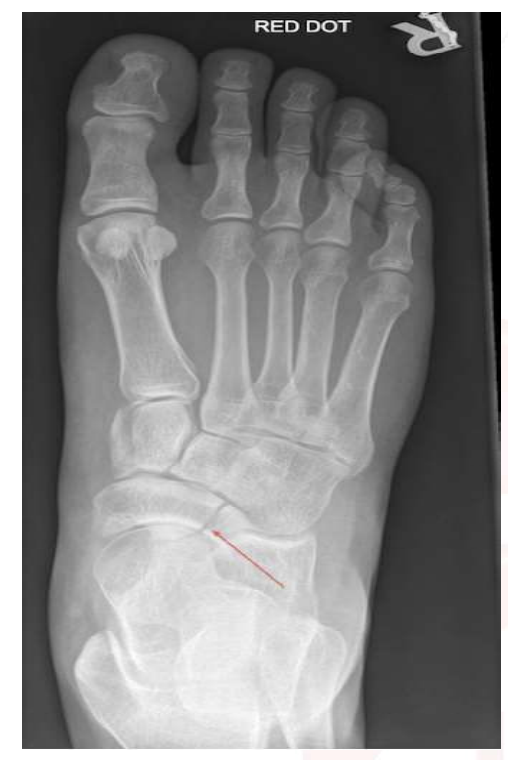

The medial cuneiform also appeared abnormal on the AP radiograph A CT scan demonstrated the calcaneo-navicular coalition fracture as seen on the plain radiographs, but also clearly delineated the incidental finding of a bipartite medial cuneiform (shown in Figure 2).

The acute fracture through the calcaneo-navicular coalition was treated non-operatively in a walking boot for a period of six weeks. During this time the pain from the calcaneo-navicular coalition fracture had resolved. At his final follow-up 4 months post-injury the patient was pain free and had returned to all normal activities.

\section{DISCUSSION}

Embryologically, the origin of both bipartite medial cuneiform and calcaneonavicular coalition is abnormal differentiation of the primordial mesenchyme in utero [1]. This case highlights an interesting process of both failure of differentiation of a single ossific nucleus, as in coalition, versus over differentiation or separation of one ossific nucleus into two segments which ultimately fail to fuse.

Whilst it is recognised that tarsal coalitions can be associated with skeletal abnormalities such as synostosis and club foot there have been very few reports of bipartite medial cuneiform associated with other skeletal abnormalities and in particular no previous description of a bipartite medial cuneiform with calcaneo-navicular coalition. This is surprising given the embryological nature of the deformity and postulated genetic predisposition [1,7]. Review of the literature demonstrated very few documented skeletal abnormalities associated with bipartite medial cuneiform; those found are summarised in Table 1. Amongst these are 3 case reports specifically describing bipartite medial cuneiform associated with another abnormality of the foot; Laffranchi et al described an archaeological case of bilateral foot polydactyly alongside a bipartite medial cuneiform [16], Burnett described fusion of an accessory calcaneus secundaris [1] and Dwight [17] and Fulwadhava [18] described two separate cases of an accessory navicular.

Table 1: Table demonstrating documented case reports of anatomical abnormalities associated with a complete bipartite medial cuneiform.

\begin{tabular}{|l|c|c|l|}
\hline \multicolumn{1}{|c|}{ Author } & Sample Type & Cases & \multicolumn{1}{|c|}{ Associated Abnormality } \\
\hline Laffranchi (2015) & Archaeological & 1 & Bilateral Foot polydactyly and dental agenesis. \\
\hline Burnett (2011) & Archaeological & 1 & $\begin{array}{l}\text { Fusion of the } 3^{\text {rd }} \text { metacarpal to the capitate + } \\
\text { accessory calcaneus secundaris. }\end{array}$ \\
\hline Dwight (1907), Fulwadhva (2007) & & 2 & Accessory navicular \\
\hline Burnett (2011) & Archaeological & 1 & Incomplete neural arches $2^{\text {nd }}-6^{\text {th }}$ sacral units \\
\hline Burnett (2011) & Archaeological & 1 & Partial sacralisation of the $5^{\text {th }}$ lumbar vertebra \\
\hline Burnett (2011) & Archaeological & 1 & Complete sacralisation $5^{\text {th }}$ lumbar vertebra \\
\hline Burnett (2011) & Archaeological & 1 & $\begin{array}{l}\text { Unilateral lunate-triquetral coalition + partially } \\
\text { bipartite squama of the Temporal bone }\end{array}$ \\
\hline Burnett (2011) & Archaeological & 1 & $\begin{array}{l}\text { Fusion } 3^{\text {rd }} \\
\text { capitate }\end{array}$ \\
\hline
\end{tabular}

The overall lack of reported cases of bipartite medical cuneiforms suggests that that it is a diagnosis which is either rare itself or rarely considered. The lack of reported associated abnormalities is unsurprising given that many of the reported cases are either from archaeological studies in which full skeletons have not been available for analysis or clinical case reports in which other anatomical variations have not been sought.

\section{CONCLUSION}

Bipartite medial cuneiform is a rare anatomical variation of the midfoot. Cases of this existing in conjunction with another anatomical anomaly of the foot are either rarely reported or considered within clinical practice. This is surprising 
given the embryological and genetic link associated with the condition. This case in particular highlights the interesting paradoxical processes of over-differentiation and failure of separation and subsequent fusion of primordial mesenchyme.

\section{Conflicts of Interests: None}

\section{REFERENCES}

[1]. Burnett SE, Caseb DT. Bipartitie medial cuneiform: New frequencies from skeletal collections and metaanalysis of previous cases. HOMO-Journal of comparative Human Biology 2011;62:109-125.

[2]. Chang GH, Chang EY, Chung CB, Resnick DL . Bipartite Medial Cuneiform: Case Report and Retrospective Review of 1000 Magnetic Resonance (MR) Imaging Studies.Case reports in Medicine 2014;1-4.

[3]. Brookes-Fazakerley SD, Jackson GE, Platt SR. An additional middle cuneiform?.Journal of surgical case reports 2015;7:1-4.

[4]. Eves TB, Mudussar AA, Oddy MA. Sports Injury to a Bipartite Medial Cuneiform in a Child.The Journal of Foot \& Ankle Surgery 2014;5:232-23.

[5]. Panu A, Konin G, Saboeiro G, Schneider R. Symptomatic Bipartite Medial Cuneiform Treated with Fluoroscopic and Ultrasound-Guided Injections. Hospital for Special Surgery 2014;10:92-97.

[6]. Elias I, Dheer S, Zoga AC. Magnetic resonance imaging findings in bipartite medial cuneiform - a potential pitfall in diagnosis of midfoot injuries: a case series. Journal of Medical Case Reports 2008;2:272.

[7]. Kjellstrom A. A case study of os cuneiforme mediale bipartum from siguna, Sweeden. International Journal of Osteoarch 2004;14:475-480.

[8]. Dellacorte MP, Parkson JL, Grisafi PJ. Bilateral medial cuneiform. Journal of the American Podiatric Medical Association.1992;82:475-478.
[9]. Azurza K, Sakellariou A. Osteosynthesis of a symptomatic bipartite medial cuneiform. Foot and ankle International 2001;22:499-501.

[10]. Chiodo CP, Parentis MA, Myerson MS. Symptomatic bipartitie medial cuneiform in an adult athlete: $A$ case report. Foot and ankle international 2002;23:348-351.

[11]. Jashashvili T, Ponce de Leo MS, Lordkipanidze D, Zollikofer CPE. First evidence of a bipartite medial cuneiform in the hominin fossil record: a case report from the Early Pleistocene site of Dmanisi. Journal of Anatomy 2010;216:705-716.

[12]. Barlow TE. Os cuneiforme 1 bipartitum. American Journal of Physical Anthropology 1942;29:95-111.

[13]. Lawrence DA, Rolen MF, Haims AH, Zayour Z, Moukaddam HA. Tarsal Coalitions: Radiographic, CT, and MR Imagining Findings. HSSJ 2014;10:153166.

[14]. Haffner M, Conklin M. Bones of the lower limb. In Bergmans comprehensive encyclopedia of human anatomic variations, Volume 1. $1^{\text {st }}$ edition. Edited by SR Tubbs, MM Shoja, M Loukas. New Jersey: WileyBlackwell; 2016:102-103.

[15]. Nalaboff KM, Schweitzer ME. MRI of tarsal coalition: frequency, distribution and innovative signs. Bulletin if the NYU Hospital for joint diseases 2008;66:14-21.

[16]. Laffranchi Z, Martin Florez JS. Foot polydactyly and bipartite medial cuneiform: A case of co-occurrence in a celtic skeleton from Verona (Italy).HOMO-Journal of comparative Human Biology 2015;66:21622.

[17]. Dwight T. Variations of the bones of the hands and feet. Philadelphia:JB Lippincott Company;1907.

[18]. Fulwadhva U, Parker RJ. Symptomatic bipartite medial cuneiform. Applied Radiology 2007;3:42-44.

How to cite this article:

Anna L Watts, Nicholas Duncan, Mark B Davies. BIPARTITE MEDIAL CUNEIFORM AND CALCANEO-NAVICULAR COALITION IN A SINGLE INDIVIDUAL. Int J Anat Res 2017;5(1):3634-3637. DOI: $10.16965 /$ ijar.2017.126 